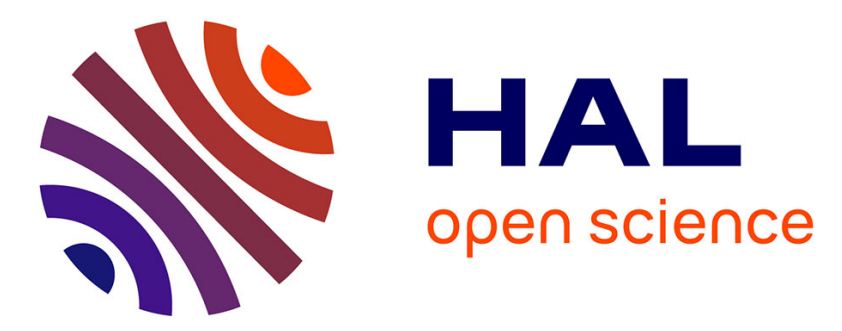

\title{
Dissipation in a sheared foam: from bubble adhesion to foam rheology
}

Sébastien Besson, Georges Debregeas, Sylvie Cohen-Addad, Reinhard Höhler

\section{To cite this version:}

Sébastien Besson, Georges Debregeas, Sylvie Cohen-Addad, Reinhard Höhler. Dissipation in a sheared foam: from bubble adhesion to foam rheology. 2008. hal-00294239v3

\section{HAL Id: hal-00294239 \\ https://hal.science/hal-00294239v3}

Preprint submitted on 17 Oct 2008

HAL is a multi-disciplinary open access archive for the deposit and dissemination of scientific research documents, whether they are published or not. The documents may come from teaching and research institutions in France or abroad, or from public or private research centers.
L'archive ouverte pluridisciplinaire HAL, est destinée au dépôt et à la diffusion de documents scientifiques de niveau recherche, publiés ou non, émanant des établissements d'enseignement et de recherche français ou étrangers, des laboratoires publics ou privés. 


\title{
Dissipation in a sheared foam: from bubble adhesion to foam rheology
}

\author{
Sébastien Besson* and Georges Debrégeas \\ Laboratoire de Physique Statistique, CNRS UMR 8550, \\ 24, rue Lhomond, 75231 Paris Cedex 05, France \\ Sylvie Cohen-Addad and Reinhard Höhler \\ Université Paris-Est, Laboratoire de Physique des Matériaux Divisés et des Interfaces, \\ UMR 8108 du CNRS, 5, boulevard Descartes, Champs-sur-Marne, 77454 Marne-la-Vallée Cedex 2, France
}

(Dated: October 14, 2008)

\begin{abstract}
The link between the rheology of 3D aqueous foam and the adhesion of neighbouring bubbles is tested by confronting experiments at two different length scales. On the one hand, the dynamics of adhesion are probed by measuring how the shape of two bubbles in contact changes as their center-to-center distance is modulated. On the other hand, the linear viscoelastic behavior of 3D foam prepared with the same soapy solution is characterized by its complex shear modulus. To connect the two sets of data we present a model of foam viscoelasticity taking into account bubble adhesion.
\end{abstract}

PACS numbers: 82.70.Rr, 47.55.D-, 83.80.Iz, 83.85.Cg

Liquid foams exhibit complex mechanical behavior $[1,2]$. When subjected to a small shear stress they respond like a linearly elastic solid, but if the applied stress is increased above the yield stress foams flow like shearthinning fluids. This behavior is related to the foam structure on the bubble scale. The elasticity is due to the reversible increase of interfacial energy induced when the bubbles are strained. Yielding and flow occur if the applied stress is large enough to trigger irreversible local bubble rearrangements $[3,4]$. In the linear regime where strain induced bubble rearragements do not exist [5] such a simple description of foam rheology would predict perfectly elastic response at frequencies sufficiently low for viscous friction to be insignificant. This is at odds with macroscopic rheological measurements of the slow linear viscoelastic response to stresses well below the yield stress. Such experiments have evidenced significant mechanical dissipation $[2,6-8]$. This behaviour has recently been explained as the consequence of the coarsening of the foam, driven by gas diffusion between neighboring bubbles [8, 9]. Indeed, coarsening induces intermittent bubble rearrangements which locally relax stress. A simple homogenization argument predicts that these relaxations yield a Maxwell liquid behaviour where the following dependence of the complex shear modulus $G^{*}$ on angular frequency $\omega$ is expected [8]:

$$
G^{*}=G^{\prime}+i G^{\prime \prime}=G_{0} \frac{i \omega \tau}{1+i \omega \tau}
$$

$G_{0}$ is the static foam elasticity that would be observed in the absence of any rearrangements. The characteristic relaxation time $\tau$ is proportionnal to the average time interval between coarsening-induced bubble rearrangements at a given place in the foam [8].

Morevoer, at frequencies $\omega>>1 / \tau$, the experimentally observed loss modulus $G$ " increases as $\omega^{1 / 2}$, indicating that here, additional relaxation processes must be active[6, 7]. Such behavior is common to several disordered close packings of small soft units such as concentrated emulsions[10] or pastes[11]. Liu et al. have proposed a generic model of viscoelasticity in these materials based on weak regions where the units (droplets, grains, bubbles... ) can slip on each other along planes whose orientation is given by the local packing structure[10]. It predicts the scaling law: $G^{*}=G_{0}\left(1+\sqrt{i \omega / \omega_{c}}\right)$ where the characteristic frequency varies with the liquid viscosity $\eta$ as $\omega_{c} \propto G_{0} / \eta$. In contrast Buzza and Cates have discussed a variety of possible origins of viscoelastic dissipation which are specific to foams and emulsions, such as surfactant transport processes and viscous friction at the gas/liquid interfaces [12]. Therefore new experimental information is needed to identify among these possible mechanisms the dominant one that governs the fast rheological response of liquid foam.

In this paper, we present experiments probing how the rheology of the liquid films is linked to the macroscopic mechanical response of foams. This is done by confronting the experimentally observed dynamic adhesive properties of bubbles to the macroscopic viscoelastic response of foams produced with the same surfactant solution. We also present a theoretical model of the coupling between interfacial film viscoelasticity and macroscopic foam viscoelasticity.

All the investigated bubbles and foams are made using a solution of tetramethyltetradecylammonium bromide diluted at a concentration of $3 \mathrm{~g} / \mathrm{l}$ in a water/glycerol mixture (volume ratio of $75 / 25$ ). To produce the foam, the solution and the gas are injected into a column filled with glass spheres as described in [13]. The gas is constituted of nitrogen saturated with perfluorohexane vapor to slow down the coarsening. We determine the liquid fraction $\Phi_{l}$ of the foam obtained at the column outlet by weighing a sample of known volume. We adjust the gas and liquid flow rates to produce foams with $\Phi_{l}=(5.0 \pm 0.2) \%$. Us- 

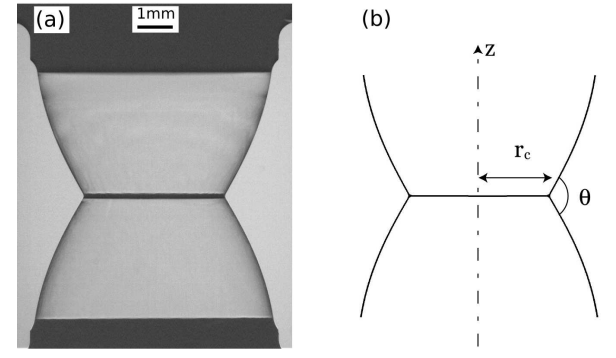

FIG. 1: (a) Image of two contacting bubbles. (b) Adhesion profile obtained after fitting the shape of the bubbles using the Young-Laplace equation. The contact radius $r_{c}$ and the contact angle $\theta$ are determined from the intersection of the reconstructed profiles.

ing diffusive light transmission, the mean bubble radius is measured: It is equal to $24 \mu \mathrm{m} \pm 2.0 \mu \mathrm{m}$ at the age of 12 minutes and $26 \mu \mathrm{m} \pm 3 \mu \mathrm{m}$ at 30 minutes. The age is the time elapsed since the instant of sample production. Pictures of the foam confined between two glass plates do not reveal any significant evolution of the polydispersity up to 40 minutes.

The adhesion between bubbles is characterized using the experimental setup described in [14]. Two hemispherical bubbles are put into contact and their profiles are recorded with a CCD camera (figure 1 (a)). Using image analysis, we measure the contact radius $r_{c}$ and the contact angle $\theta$ formed at the junction between the two bubbles (figure $1(\mathrm{~b})) . r_{c}$ and $\theta$ are monitored as a function of time $t$.

Macroscopic foam viscoelasticity is investigated using a rheometer (Bohlin, CVOR 150) equipped either with a plate/plate geometry (plate radius equal to $3 \mathrm{~cm}$, gap of $3 \mathrm{~mm}$ ) or with a cone/plate geometry ( cone radius $=3$ $\mathrm{cm}$, cone angle $\left.=4^{\circ}\right)$. For both geometries, the surfaces in contact with the foam are grooved to avoid wall slip. All along the experiment, the air in contact with the sample in the rheometer is saturated with humidity. To erase the memory of the strain applied to the sample during the filling of the rheometer, the sample is pre-sheared by an oscillating strain of frequency $1 \mathrm{~Hz}$ and amplitude $\varepsilon=10^{-1}$, close to the yield strain. This preshear is applied at a foam age of 3.5 minutes and lasts five minutes. The rheological measurements start at a foam age of $12 \mathrm{~min}$ and do not last more than 10 minutes.

The results of the dynamic bubble adhesion study have been reported in detail in a recent paper [14]. The distance between the bubble supports is modulated in time along the z-direction. We find that a sinusoidal modulation induces a sinusoidal variation of the contact radius $r_{c}(t)=r_{c 0}+\Delta r_{c} e^{i \omega t}$ accompanied by an oscillation of the contact angle around its static value $\theta_{\text {stat }}$ such that $\theta(t)=\theta_{\text {stat }}+\Delta \theta(t) e^{i \omega t}$. The amplitude of the oscillation is found to be linearly related to the amplitude of the relative variation of the contact radius $\Delta r_{c} / r_{c 0}$. To describe

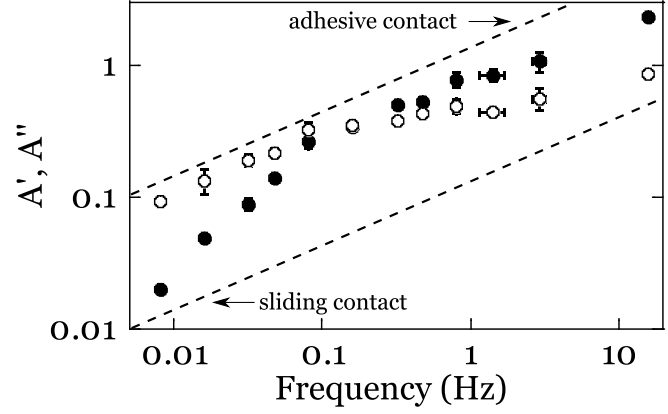

FIG. 2: Evolution with frequency of the angular moduli $A^{\prime}(\bullet$ ) and $A^{\prime \prime}$ (०) defined in Eq. 2. The dashed lines correspond to the predictions of the moduli for the two limiting cases of adhesive or sliding contacting bubbles.

this relation as well as the phase shift between the contact radius and the contact angle oscillations, we introduce the complex modulus $A^{*}$ whose real and imaginary parts, $A^{\prime}$ and $A^{\prime \prime}$, describe the in-phase and out-of-phase angular response:

$$
\Delta \theta(t)=\frac{\Delta r_{c}}{r_{c 0}}\left(A^{\prime}+i A^{\prime \prime}\right)
$$

The evolution of $A^{\prime}$ and $A^{\prime \prime}$ with frequency is compared to that expected for either adhesive or sliding bubbles which both scale as the square root of the frequency (figure 2). It has been interpreted as the consequence of surfactant transport between the bulk of the film and the interfaces as well as along the interfaces [14].

In the linear regime, the frequency and temporal responses of a viscoelastic material provide equivalent information. Since creep experiments are more convenient for probing the slow rheological response, we use this test to study our foam samples over time intervals chosen short enough so that the evolutions of bubble size and bubble rearrangement rate are negligible. After the preshear, at an age of $12 \mathrm{~min}$, we apply to the foam a shear stress step of amplitude $\sigma_{0}$, chosen small enough for the response to be linear and to avoid yielding. The induced strain $\varepsilon(t)$ is measured as a function of time, and the response is decribed in terms of the compliance $J(t)=\varepsilon(t) / \sigma_{0}$ (figure 3 ). The data obtained using cone/plate and plate/plate geometries are consistent. The asymptotically linear increase of $J(t)$ with time is consistent with the Maxwell model Eq. 1 that predicts the following compliance :

$$
J(t)=\frac{1}{G_{0}}\left(1+\frac{t}{\tau}\right)
$$

The best fit to the data measured for $t>28 \mathrm{~s}$ yields $G_{0}=206 P a \pm 4 P a$ and $\tau=246 s \pm 10 s$. The transitory response for $t<28 \mathrm{~s}$ corresponds to a second slow relaxation probably governed by the intrinsic viscosity of the interfaces [8]. To simplify, we will neglect it since its contribution to $G^{*}$ above $1 \mathrm{~Hz}$ is small. 


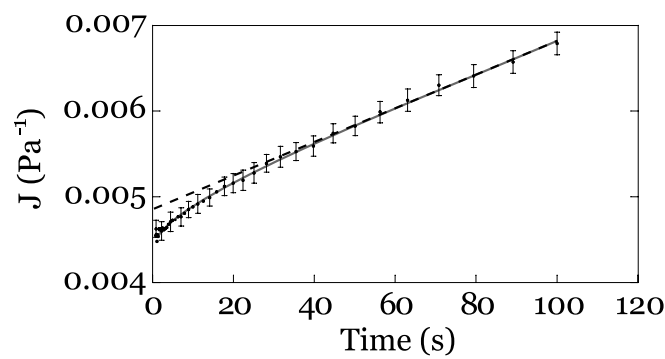

FIG. 3: Time evolution of the compliance during a creep experiment with a constant stress $\sigma_{0}$ applied during $100 \mathrm{~s}$. The compliance is averaged over 4 samples and 3 values of $\sigma_{0}$ comprised between $3 \mathrm{~Pa}$ and $6 \mathrm{~Pa}$. The dashed line corresponds to the prediction of Eq. (3) (see text).

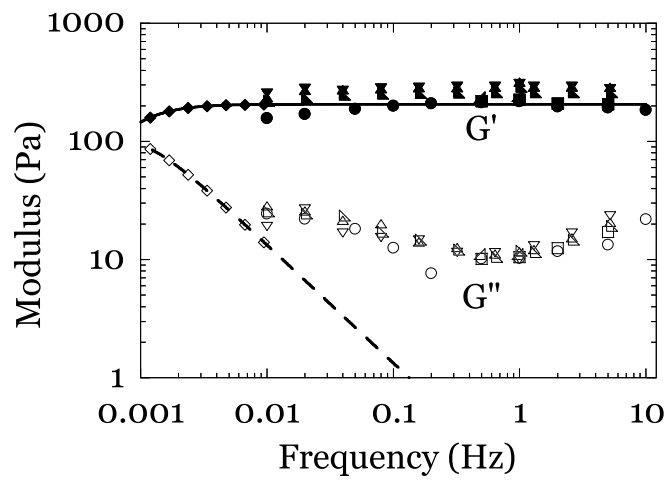

FIG. 4: Frequency dependence of $G^{\prime}$ (closed symbols) and $G$ " (open symbols) measured for 6 samples. The diamonds are deduced from the creep data using Laplace transform while the other symbols correspond to oscillatory data. The lines represent the moduli predicted by (Eq. 1) with $G_{0}=206 P a$ and $\tau=246 s$.

Moreover, the viscoelastic response is probed using an oscillatory test in a frequency range between $0.01 \mathrm{~Hz}$ and $10 \mathrm{~Hz}$. After the preshear, at an age of $12 \mathrm{~min}$, a sinusoidal shear strain of amplitude 0.01 much smaller than the yield strain (of the order of 0.1) is applied and the complex modulus $G^{*}$ is measured. The data obtained using cone/plate and plate/plate geometries are consistent. Figure 4 shows the elastic and viscous moduli measured as a function of frequency. It also shows the moduli deduced using Eq. 1 with the values of $G_{0}$ and $\tau$ obtained in the creep experiments (FIG. 3). These data are limited to frequencies below $0.01 \mathrm{~Hz}$ because the step stress response exhibits inertio-elastic vibrations at short times. The elastic modulus is observed to be almost constant over the whole range of frequencies while the loss modulus goes through a minimum around $1 \mathrm{~Hz}$. The difference between the measured $G^{\prime \prime}$ and the Maxwell one means that other relaxation processes must be taken into account.

To link the measured bubble adhesion to bulk foam rheology, an analysis at the scale of the film network is needed. We start from the model initially developed by
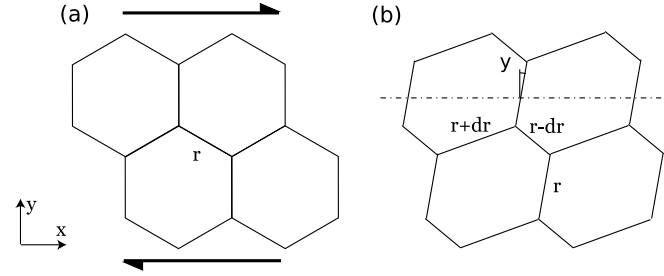

FIG. 5: The Princen model for the deformation of a 2D hexagonal dry foam. (a) Initial structure. (b) After a small quasistatic shear strain has been applied in the $x$ direction, the angles formed at the vertices remain equal to $120^{\circ}$.

Princen for dry 2D foams [3]. The foam is simply pictured as a regular 2D hexagonal lattice (figure 5(a)) obeying Plateau's rule which imposes a $120^{\circ}$ angle between films meeting at vertices. Moreover, each cell surface is conserved when the foam is sheared, as if the gas contained in the bubbles were incompressible. Princen calculated how the orientations of the different films are modified by an imposed static shear strain $\varepsilon$. For the structure shown in figure 5(b) he found that to first order, the edges perpendicular to the shear direction turn by an angle $\psi=\varepsilon / 2$. The surface tension forces of the films crossing the horizontal dashed line in the figure and the spacing between these films determines the macroscopic shear stress to first order as $\sigma=2 \gamma \psi /(\sqrt{3} r)$ where $\gamma$ is the surface tension of the liquid gas interface. Thus, Princen predicted the static shear modulus $G_{0}=\sigma / \varepsilon=\gamma /(\sqrt{3} r)$. Under dynamic conditions this relation remains unchanged but the angles between films that meet at vertices no longer follow Plateau's rule. The direction of the films initially perpendicular to the shear direction is now described by the angle $\psi+\delta \psi$ where $\delta \psi$ is given by equation 2 [14], by analogy with the double-bubble experiments. On this basis, the complex shear modulus can be estimated as a function of the complex angular modulus $A^{*}$ :

$$
G^{*}=G_{0}\left[1+\alpha A^{*}\right]
$$

$\alpha$ is a geometrical constant, equal to $\sqrt{3}$ in the case of the $2 \mathrm{D}$ hexagonal dry film network.

To compare our model to the measured rheological data, we consider $\alpha$ as an adjustable parameter since its value for a disordered 3D wet foam is difficult to predict theoretically. Moreover, for such a comparison one must also take into account the slow relaxation due to coarsening- induced bubble rearrangements. It has been shown that these rearrangements can be modelized as zones that are dispersed throughout an elastic matrix and that temporarily loose their rigidity [8]. A homogenization argument on this basis leads to the Maxwellian viscoelastic behavior described by Eq. 1. The kind of homogenization used here can be generalized from elastic to viscoelastic matrices [15]. The viscoelasticity due to bubble adhesion can therefore be taken into account by replacing in Eq. (1) $G_{0}$ by the complex shear modulus 


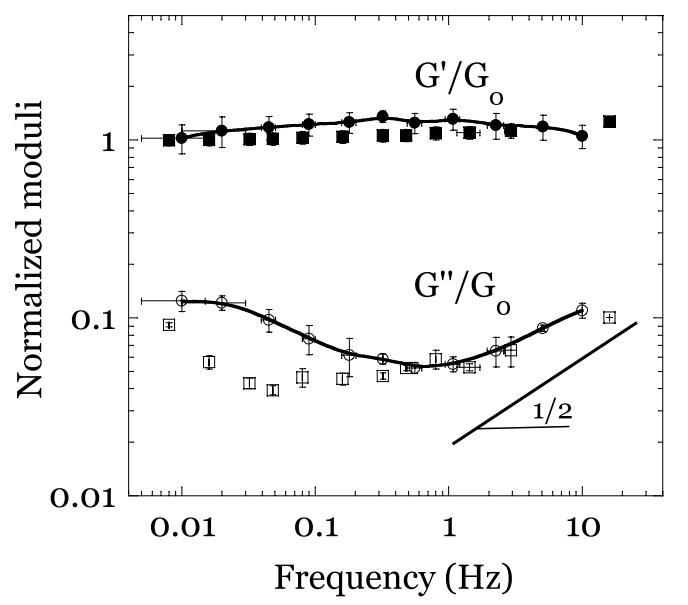

FIG. 6: Comparison of measured and predicted evolutions with frequency of the normalized elastic and loss moduli : The measured data of figure $4\left(G^{\prime}: \bullet, G^{\prime \prime}: \circ\right)$ are normalized by $G_{0}$. The prediction ( $G^{\prime}: \mathbf{\square}, G^{\prime \prime}: \square$ ) of Eq. (5) with $G_{0}=$ $206 P a, \tau=246 s$ and $\alpha=0.12$ is based on the experimental angular moduli of figure 2 . The lines are guides to the eye.

predicted by Eq. (4):

$$
\frac{G^{*}}{G_{0}}=\frac{i \omega \tau}{1+i \omega \tau}\left[1+\alpha A^{*}\right]
$$

Figure 6 establishes the link between the macroscopic shear modulus data and the prediction of our model based on the two-bubble response (Eq. (5)). The elastic and loss moduli (showed in figure 4) are normalized by the value of $G_{0}$ obtained from the creep experiment, and averaged over results for several samples. The moduli deduced from the two-bubble measurements are predicted using Eq. (5). The parameter $\alpha$ is set to 0.12 which provides the best fit between the two sets of data. The good agreement between all the data at high frequency presented in figure (6) shows that the viscoelasticity in this regime can be accounted for by the dissipation due to surfactant transport in the liquid films. The discrepancy between the predicted and measured dissipation at frequencies between $0.01 \mathrm{~Hz}$ and $0.1 \mathrm{~Hz}$ suggests that here, relaxation processes not yet taken into account in the model are active, possibly related to intrinsic dilationnal interfacial viscosity as evidenced in previous creep measurements [8].

Moreover, the difference between the theoretical value $\alpha=\sqrt{3}$, and the fitted one, 0.12 , underlines the limitations of the proposed model. First, the Princen model describes $2 \mathrm{D}$ foams while our experiments probe $3 \mathrm{D}$ foam. Secondly, the lattice considered in the model is ordered, in contrast to the experimentally investigated foam. As a perspective for further work, we note that at frequencies so high that surfactant diffusion along the interfaces becomes insignificant on the time scale of an oscillation, one expects the complex angular modulus $A^{*}$ to be proportional to the film dilational modulus $E^{*}$ [14]. Indeed, if the interfacial elasticity is limited by the diffusion of the surfactant from the bulk towards the interface, its dilatational modulus $E^{*}$ is given by the Lucassen van den Tempel model. In the limit where surfactants have time to diffuse from the bulk to the surface, we expect $[16,17]: E^{*} \sim E_{0} \sqrt{i \omega \tau_{d}} . E_{0}$ is the limit elasticity and $\tau_{d}$ is a characteristic surfactant diffusion time. As a consequence the complex shear modulus of foams at high frequency should scale as the square root of frequency. Such a frequency dependency has indeed been observed in aqueous foams up to $60 \mathrm{~Hz}$ by Gopal et al. [7]. Thus interfacial dilatational viscoelasticity as taken into account in our model predicts the same scaling with frequency of the complex shear modulus as the model based on relaxations in generic weak regions mentionned above[10]. To conclude, we present a model that sucessfully predicts the fast linear viscoelastic response of bulk foam on the basis of experiments probing the interfacial rheology at the scale of two bubbles in contact. These findings prepare the ground for a future model of foam rheology that fully captures how local structure and physicochemical behavior are coupled to the macroscopic response of foams and similar complex fluids.

* Electronic address: sbesson@oeb.harvard.edu

[1] D. Weaire and S. Hutzler, The Physics of Foams (Oxford University Press, New York, 1999).

[2] R. Höhler and S. Cohen-Addad, J. Phys.: Condens. Matter 17, R1041 (2005).

[3] H. M. Princen, J. Colloid Interface Sci. 91, 160 (1983).

[4] A. Kabla and G. Debrégeas, J. Fluid Mech. 587, 23 (2007).

[5] R. Höhler, S. Cohen-Addad, and H. Hoballah, Phys. Rev. Lett. 79, 1154 (1997).

[6] S. Cohen-Addad, H. Hoballah, and R. Höhler, Phys. Rev. E 57, 6897 (1998).

[7] A. Gopal and D. Durian, Phys. Rev. Lett. 91, 188303 (2003).

[8] S. Cohen-Addad, R. Höhler, and Y. Khidas, Phys. Rev. Lett. 93, 028302 (2004).

[9] S. Vincent-Bonnieu, R. Höhler, and S. Cohen-Addad, Europhys. Lett. 74, 533 (2006).

[10] A. J. Liu, S. Ramaswamy, T. G. Mason, H. Gang, and D. A. Weitz, Phys. Rev. Lett. 76, 3017 (1996).

[11] C. Derec, G. Ducouret, A. Ajdari, and F. Lequeux, Phys. Rev. E 67, 061403 (2003).

[12] D. Buzza, C.-Y. Lu, and M. Cates, J. Phys. II 5, 37 (1995).

[13] F. Rouyer, S. Cohen-Addad, M. Vignes-Adler, and R. Höhler, Phys. Rev. E 67, 021405 (2003).

[14] S. Besson and G. Debrégeas, Eur. Phys. J. E 24, 109 (2007).

[15] J. F. Palierne, Rheol. Acta 29, 204 (1990).

[16] J. Lucassen and M. Van den Tempel, J. Colloid Interface Sci. 41, 491 (1972).

[17] J. Lucassen, Faraday Discussions of the Chemical Society 59, 76 (1975). 